\title{
Criminologie
}

\section{Du culte des héros à la concurrence des victimes}

\section{Jean-Michel Chaumont}

Volume 33, numéro 1, printemps 2000

La victimologie : quelques enjeux

URI : https://id.erudit.org/iderudit/004712ar

DOI : https://doi.org/10.7202/004712ar

Aller au sommaire du numéro

\section{Éditeur(s)}

Les Presses de l'Université de Montréal

ISSN

0316-0041 (imprimé)

1492-1367 (numérique)

Découvrir la revue

\section{Citer cet article}

Chaumont, J.-M. (2000). Du culte des héros à la concurrence des victimes. Criminologie, 33(1), 167-183. https://doi.org/10.7202/004712ar

\section{Résumé de l'article}

Dans un premier temps, l'auteur résume l'histoire d'une attente de reconnaissance frustrée dans l'immédiat après-guerre : celle des survivants de la Shoah. Ceux-ci subirent en effet un processus de victimisation secondaire analogue à ceux que l'on observe auprès d'autres catégories de victimes, telles les victimes de viol. Non seulement leur expérience spécifique, en tant que Juifs, fut occultée mais ils furent en outre doublement stigmatisés pour leurs réactions face à l'entreprise criminelle des nazis : d'une part, les Juifs assassinés se seraient laissé conduire à la mort « comme des moutons à l'abattoir "; d'autre part, les survivants auraient adopté des comportements indignes pour survivre. Alors que les déportés résistants recueillaient tous les lauriers de la gloire, les survivants juifs furent confinés dans la honte. Leurs légitimes attentes de reconnaissance restèrent ainsi longtemps insatisfaites. Ils n'eurent pas droit aux statuts honorifiques et, bien souvent, ne furent même pas mentionnés explicitement sur les monuments commémoratifs aux victimes du Troisième Reich. Il en résulta des traumatismes durables dont les conséquences se sont manifestées ultérieurement.

Pendant près de vingt ans en effet, cette situation perdura sans susciter beaucoup de protestations. Au milieu des années 1960 cependant, une réaction s'amorça et une entreprise de réhabilitation, voire de glorification, des victimes fut menée avec succès. Le stigmate fut effacé, souvent même inversé : être un survivant de la Shoah est devenu un titre prestigieux. Le moyen détourné de cette revalorisation spectaculaire a été la revendication polémique de la singularité absolue de la Shoah : son unique uniqueness.

Cette manière indirecte de mener une authentique lutte pour la reconnaissance a néanmoins engendré des effets pervers notables à de multiples points de vue. L'auteur évoque plus particulièrement celui de « la concurrence des victimes ». À partir du moment où un groupe prétend que sa victimisation est sans aucune commune mesure avec d'autres persécutions, il est prévisible que d'autres victimes du nazisme ou d'autres tragédies historiques s'insurgent et soutiennent le contraire. D'où, depuis un quart de siècle, l'émergence de débats sordides et interminables sur la comparabilité des crimes et des souffrances. Les oppositions se déplacent et les victimes luttent entre elles plutôt que de faire front commun. La réhabilitation morale des morts et des survivants se dégrade alors en une lutte pour la distinction sociale attisée par un contexte général où le statut de victime est devenu un passe-droit enviable.

En conclusion, l'auteur s'interroge précisément sur les raisons et les causes qui ont conduit à cette valorisation remarquable du statut de victime au cours des dernières décennies. Tant les victimes de faits divers que les victimes de tragédies historiques en profitent. Un changement socioculturel majeur s'est ainsi produit dont il reste à élucider la dynamique. L'émergence de la victimologie comme discipline autonome en constitue probablement une des manifestations et c'est pourquoi la question ne saurait laisser les victimologues indifférents. Jusqu'il y a peu, les héros méritaient d'être rétribués, tant matériellement que symboliquement, pour ce qu'ils avaient accompli. Il semble qu'à présent ce soient avant tout les victimes qui méritent d'être compensées pour ce qu'elles ont subi. Comment expliquer cette évolution remarquable des sensibilités collectives ? La revalorisation concomitante du statut des victimes de l'Histoire et des victimes de faits divers incite à chercher des réponses qui transcendent les différences de ces catégories tellement hétérogènes. L'auteur suggère à titre hypothétique de lier la promotion du statut de victime au redéploiement de la question sociale dans les sociétés occidentales : si, comme certains l'affirment, le conflit central n'est plus celui de l'intégration mais celui de l'insertion, la posture de la victime pourrait bien représenter la posture revendicatrice privilégiée. Il resterait alors à en tracer les potentialités et les représe
Ce document est protégé par la loi sur le droit d'auteur. L’utilisation des services d’Érudit (y compris la reproduction) est assujettie à sa politique d'utilisation que vous pouvez consulter en ligne.

https://apropos.erudit.org/fr/usagers/politique-dutilisation/ 


\title{
Du culte des héros à la concurrence des victimes
}

\author{
Jean-Michel Chaumont \\ Chercheur qualifié du Fonds National de la Recherche Scientifique \\ Unité d'Anthropologie et de Sociologie \\ Université catholique de Louvain • Belgique \\ chaumont@anso.ucl.ac.be
}

RÉSUMÉ - Dans un premier temps, l'auteur résume l'histoire d'une attente de reconnaissance frustrée dans l'immédiat après-guerre : celle des survivants de la Shoah. Ceux-ci subirent en effet un processus de victimisation secondaire analogue à ceux que l'on observe auprès d'autres catégories de victimes, telles les victimes de viol. Non seulement leur expérience spécifique, en tant que Juifs, fut occultée mais ils furent en outre doublement stigmatisés pour leurs réactions face à l'entreprise criminelle des nazis : d'une part, les Juifs assassinés se seraient laissé conduire à la mort « comme des moutons à l'abattoir »; d'autre part, les survivants auraient adopté des comportements indignes pour survivre. Alors que les déportés résistants recueillaient tous les lauriers de la gloire, les survivants juifs furent confinés dans la honte. Leurs légitimes attentes de reconnaissance restèrent ainsi longtemps insatisfaites. Ils n'eurent pas droit aux statuts honorifiques et, bien souvent, ne furent même pas mentionnés explicitement sur les monuments commémoratifs aux victimes du Troisième Reich. Il en résulta des traumatismes durables dont les conséquences se sont manifestées ultérieurement.

Pendant près de vingt ans en effet, cette situation perdura sans susciter beaucoup de protestations. Au milieu des années 1960 cependant, une réaction s'amorça et une entreprise de réhabilitation, voire de glorification, des victimes fut menée avec succès. Le stigmate fut effacé, souvent même inversé : être un survivant de la Shoah est devenu un titre prestigieux. Le moyen détourné de cette revalorisation spectaculaire a été la revendication polémique de la singularité absolue de la Shoah : son unique uniqueness.

Cette manière indirecte de mener une authentique lutte pour la reconnaissance a néanmoins engendré des effets pervers notables à de multiples points de vue. L'auteur évoque plus particulièrement celui de «la concurrence des victimes ». À partir du moment où un groupe prétend que sa victimisation est sans aucune commune mesure avec d'autres persécutions, il est prévisible que d'autres victimes du nazisme ou d'autres tragédies historiques s'insurgent et soutiennent le contraire. D'où, depuis un 
quart de siècle, l'émergence de débats sordides et interminables sur la comparabilité des crimes et des souffrances. Les oppositions se déplacent et les victimes luttent entre elles plutôt que de faire front commun. La réhabilitation morale des morts et des survivants se dégrade alors en une lutte pour la distinction sociale attisée par un contexte général où le statut de victime est devenu un passe-droit enviable.

En conclusion, l'auteur s'interroge précisément sur les raisons et les causes qui ont conduit à cette valorisation remarquable du statut de victime au cours des dernières décennies. Tant les victimes de faits divers que les victimes de tragédies historiques en profitent. Un changement socioculturel majeur s'est ainsi produit dont il reste à élucider la dynamique. L'émergence de la victimologie comme discipline autonome en constitue probablement une des manifestations et c'est pourquoi la question ne saurait laisser les victimologues indifférents. Jusqu'il y a peu, les héros méritaient d'être rétribués, tant matériellement que symboliquement, pour ce qu'ils avaient accompli. Il semble qu'à présent ce soient avant tout les victimes qui méritent d'être compensées pour ce qu'elles ont subi. Comment expliquer cette évolution remarquable des sensibilités collectives? La revalorisation concomitante du statut des victimes de l'Histoire et des victimes de faits divers incite à chercher des réponses qui transcendent les différences de ces catégories tellement hétérogènes. L'auteur suggère à titre hypothétique de lier la promotion du statut de victime au redéploiement de la question sociale dans les sociétés occidentales : si, comme certains l'affirment, le conflit central n'est plus celui de l'intégration mais celui de l'insertion, la posture de la victime pourrait bien représenter la posture revendicatrice privilégiée. Il resterait alors à en tracer les potentialités et les limites.

ABSTRACT - In this article, the author first summarizes the frustrated expectations of survivors of the shoah immediately following World War II. Survivors, expecting recognition of their experience, in fact experienced a process of secondary victimization comparable to that of another category of victims, survivors of sexual assault. Not only were their individual experiences as Jews obscured, they were doubly stigmatized for their reaction to the criminal enterprise of the Nazis. On the one hand, murdered Jews had allowed themselves to be killed "like lambs to the slaughter" ; on the other, survivors had adopted undignified means in order to survive. Thus, while deported resisters gathered laurels, Jewish survivors were covered with shame.

Their legitimate expectations of recognition went unmet for many years. Not meriting an honourable status, they were often denied explicit mention in monuments commemorating victims of the Third Reich. This treatment resulted in an enduring trauma, the consequences of which were not manifested until many years later.

This state of affairs in fact continued for almost twenty years without raising significant objections. During the 1960s, however, a reaction set in and a rehabilitative effort to wit, a glorification of the victims - was successfully conducted. Stigmata were erased and even reversed : to be a survivor of the Shoah became a mark of prestige. The convoluted means by which this spectacular about-face occurred was a polemic vindication of an absolute incomparability, the "unique uniqueness", of the Shoah.

The indirect means of conducting a legitimate struggle for recognition nevertheless generated some negative effects, most notable of which is the "competition of victims". 
From the moment that one group claims a victimization not comparable to the persecution of another, it may be expected that other victims of Nazism and other historical tragedies will arise and maintain the contrary. Thus, for a quarter of a century, there has been a sordid and interminable debate with regard to the comparability of crimes and suffering. The results is opposition, with victims struggling with each other instead of creating a common front. The moral rehabilitation of the dead and of survivors is reduced to a struggle for social distinction, within a general context where victim status has the enviable status of a claim for rights.

By way of conclusion, the author questions the precise reasons and causes that have resulted in the remarkable enhancement of victim status in recent decades. This change has benefited victims of various other types of acts as much as victims of historical tragedies. A major socio-cultural shift has resulted, the dynamics of which remain to be elucidated. The emergence of victimology as a separate discipline is probably one of its manifestations, and victimologists cannot be indifferent to this issue.

Until recently, heroes deserved compensation, material as well as symbolic, for their accomplishments. It appears at present that victims, more than any other group, deserve to be compensated for their sufferings. How can this remarkable shift in the collective consciousness be explained? The resulting revalidation of historical and incidental victim status inspires a search for answers that transcend such heterogenic categories. The author hypothesizes a connection between the promotion of the victim status and the reformulation of a question common to western societies: if, as affirmed by some, the central conflict is not that of integration but that of insertion, the privileged position of the victim may well be justified. Its potential and limits have yet to be examined.

RESUMEN - El autor resume la historia de una aspiración al reconocimiento que se verá frustrada en el periodo inmediato a la Segunda Guerra Mundial : la de los sobrevivientes de la Shoah, quienes serán efectivamente objeto de un proceso de victimización secundaria análogo al que puede observarse en otras categorías de víctimas, como es el caso de las víctimas de violación. Así, no sólo terminó ocultándose su experiencia específica como judíos, sino que fueron doblemente estigmatizados debido a sus reacciones frente a la empresa criminal de los nazis : por una parte, los judíos asesinados se habrían dejado conducir a la muerte « como reses al matadero », y, por otra, los sobrevivientes habrían adoptado comportamientos considerados como indignos, a fin de sobrevivir. Mientras que los deportados que resistieron recibirían los laureles de la gloria, los sobrevivientes judíos habrían de ser confinados a la vergüenza. Su legítima aspiración al reconocimiento quedaría insatisfecha durante largo tiempo. El derecho a alcanzar rangos honoríficos les sería negado e incluso, no pocas veces, se dejaría de mencionar expresamente sus nombres en los monumentos conmemorativos erigidos a las víctimas del Tercer Reich. De todo ello resultaron traumas profundos, cuyas consecuencias se manifestaron con posterioridad.

En efecto, durante casi veinte años esta situación perduraría sin levantar mayores protestas. No obstante, a mediados de los años 1960 se observa una reacción, a la que seguiría una verdadera tarea de rehabilitación e incluso de glorificación de las víctimas que habría de concluir con éxito. De esta manera, les fue borrado el estigma, el cual a 
menudo resultó invertido : ser un sobreviviente de la Shoah llegó a convertirse en un título de prestigio. El medio para lograr esta revalorización espectacular lo constituyó la reivindicación polémica del carácter singular absoluto que envuelve la Shoah, es decir : su singularidad única.

Esta manera especial de llevar a cabo una lucha auténtica por el reconocimiento habría de producir sin embargo efectos indeseables de importancia, y ello desde distintos puntos de vista. El autor evoca particularmente el de la « competición entre las víctimas ». A partir del momento en que un grupo pretende que su victimización resulta incomparable a la de otras persecuciones, era perfectamente previsible que otras víctimas del nazismo o de otras tragedias históricas protestaran y sostuvieran lo contrario. De allí y desde hace un cuarto de siglo, han aparecido interminables y sórdidos debates sobre la comparabilidad de los crímenes y del sufrimiento. De esta manera, las oposiciones se desplazan y las víctimas luchan entre sí, en lugar de formar un frente común. La rehabilitación moral de los muertos, así como la de los sobrevivientes se deteriora, transformándose en una lucha por la distinción social estimulada por un contexto general en el que la condición de víctima se convierte en un favor envidiable.

Como conclusión, el autor se interroga claramente sobre las razones y las causas que han conducido a esta valorización particular de la condición de víctima en el transcurso de las últimas décadas, y de la cual se han beneficiado tanto las víctimas de hechos diversos como las víctimas de tragedias históricas. Se ha producido entones un cambio estructural mayor cuya dinámica no ha sido hasta ahora totalmente aclarada. La emergencia de la victimología como disciplina autónoma constituye probablemente una de sus manifestaciones, por lo que puede suponerse que tales cuestiones no han de dejar indiferentes a los victimólogos. Hasta hace poco, los héroes merecían ser retribuidos tanto en lo material como simbólicamente, en razón de lo que habian cumplido. En la actualidad, serían particularmente las víctimas las que merecen ser compensadas por sus sufrimientos. ¿Cómo explicar esta importantísima evolución en la sensibilidad colectiva? La revalorización concomitante de la condición de víctimas de la Historia y de víctimas de hechos diversos incita a la búsqueda de respuestas que trasciendan las diferencias entre categorias tan disímiles. El autor propone a título de hipótesis vincular la promoción de la condición de víctima al replanteamiento de la cuestión social en las sociedades occidentales : si, como lo afirman algunos, el conflicto central no es ahora el de la integración sino el de la inserción, la situación de la víctima bien podría constituir la posición reivindicatoria privilegiada. Quedarían por delinear sus potencialidades y sus limitaciones. 


\section{Introduction}

On n'oublie pas que l'histoire de chacun se fait à travers le besoin d'être reconnu sans limite ; l'amitié

désigne cette capacité infinie de reconnaissance.

Imaginer que ce besoin soit constamment celui d'autrui, que l'autre comme nous-même soit livré à cette exigence et acharné à obtenir réponse, qu'il se dévore lui-même et qu'il soit comme une bête si la réponse ne vient pas, c'est à quoi on devrait s'obliger

et c'est l'enfer de la vie quand on y manque. Robert Antelme (1958 : 113)

Même si je ne suis pas criminologue, il est compréhensible qu'une recherche - issue de plusieurs années de travail au sein de la Fondation Auschwitz de Bruxelles - ayant finalement abouti à la publication d'un livre intitulé La concurrence des victimes (Chaumont, 1997) puisse intéresser un public spécialisé en victimologie. Il me faut cependant préciser qu'avant d'être un livre, ce manuscrit fut une thèse dont le titre, plus docte, était Connaissance ou reconnaissance? Les enjeux du débat sur la singularité de la Shoab (Chaumont, 1995). Entre les deux intitulés, la distance semble énorme alors que le propos est pourtant strictement identique, mais le titre originel fait référence au point de départ de la recherche tandis que La concurrence des victimes met davantage en exergue un de ses points d'arrivée. Il faut bien avouer toutefois qu'entre les deux, il y a un long chemin qui est celui du destin tortueux de l'attente de reconnaissance des victimes de la Shoah. Ma principale hypothèse fut en effet que l'enjeu véritable de la revendication de l'unicité de la Shoah était la satisfaction longtemps différée d'une attente de reconnaissance frustrée dans les années d'après-guerre et je me suis efforcé de montrer par quels détours surprenants une lutte pour la reconnaissance sociale d'un groupe de personnes discriminées avait emprunté les voies d'un pseudo-débat scientifique sur la connaissance historique d'un événement.

Je présume que pour le lectorat de cette revue, l'analyse de la polémique sur l'unicité de la Shoab est d'un intérêt secondaire et je vais donc davantage m'attacher ici à résumer dans ses grandes lignes mon interprétation des tenants et aboutissants de la lutte pour la reconnaissance qui lui est sous-jacente. Ce faisant, je m'attarderai sur ce qui me semble représenter un des effets pervers principaux de la polémique, à savoir la création de conflits artificiels entre groupes de victimes. En 
guise de conclusion, je ferai part d'une question, nullement originale mais qui demeure, à mon avis, irrésolue alors même que le sens de l'activité des victimologues en est largement tributaire.

Au préalable, un mot cependant afin de prévenir des malentendus fréquents dès que l'on aborde un sujet aussi sensible : en ce qui me concerne, je suis convaincu que la Shoab fut un événement majeur du millénaire précédent et que le judéocide présente incontestablement de nombreux traits singuliers. Mais je ne crois pas pour autant, comme le soutiennent à des titres divers les tenants de la thèse de l'unicité absolue de l'événement, qu'il s'agisse d'un phénomène historique totalement incomparable à d'autres facettes de la criminalité nazie ou à des crimes contre l'humanité et génocides perpétrés en d'autres lieux et en d'autres temps. Mon propos n'est toutefois pas d'entrer ici dans le détail des discussions sur la comparabilité et bien plutôt de porter le débat au niveau d'un des enjeux latents, mais déterminants, de la polémique : celui des attentes de reconnaissance des victimes. Il en est d'autres, telles par exemple les récupérations politiques de l'événement par différents acteurs, notamment l'État d'Israël, qui sont bien documentées par ailleurs et n'appartiennent pas à mon champ d'étude.

\title{
Le singulier destin d'une attente de reconnaissance frustrée
}

\author{
Les " héros » et les autres...
}

La France entière se réjouit ou fraternise dans les rues, les luttes sociales semblent provisoirement oubliées ; les journaux consacrent des colonnes entières aux prisonniers de guerre, aux déportés.

Va-t-on parler des Juifs? Va-t-on saluer le retour parmi nous de ces rescapés, va-t-on donner une pensée à ceux qui sont morts dans les chambres à gaz de Lublin? Pas un mot. Pas une ligne dans les quotidiens. C'est qu'il ne faut pas irriter les antisémites. Jean-Paul Sartre (1946: 93).

Il est désormais bien établi par la recherche historique que le retour des déportés ne fut pas le même pour toutes les catégories de victimes de la criminalité national-socialiste (Wieviorka, 1992 ; Lagrou, 1998). Tous étaient certes objets de commisération mais seuls certains étaient aussi 
objets d'admiration : les femmes et les hommes qui, par conviction patriotique ou antifasciste, avaient enduré l'enfer concentrationnaire en raison de leur lutte contre les nazis. Numériquement les plus nombreux parmi les rescapés - l'écrasante majorité des déportés juifs ou " raciaux » (comme on disait alors) avait été assassinée dans les chambres à gaz -, les anciens résistants furent les bénéficiaires presque exclusifs de la reconnaissance publique. À l'époque, pour toutes sortes de raisons qu'il serait trop long de détailler ici, cet état de fait ne suscita que de rarissimes réactions. Citons néanmoins ce cri du cœur d'un rescapé d'Auschwitz en 1946 :

Notre peuple juif vient de vivre le chapitre le plus cruel de son histoire. Six millions de Juifs sont tombés! Ils n'avaient rien fait pour mériter ce sort! La moindre chose que nous, survivants, pouvons demander, c'est qu'on reconnaisse les sacrifices que nous et nos morts avons faits! Nous ne demandons que de nous concéder, à nous également, une modeste petite place au soleil (Klieger, $1946: 191)$.

Non seulement il ne fut pas entendu mais, à l'inverse, une chape de silence recouvrit l'expérience spécifique des déportés juifs qui fut rendue largement invisible par son amalgame avec l'expérience générique des «victimes de la barbarie fasciste ». Symbole aujourd'hui à peine croyable de cette longue période d'occultation généralisée de l'identité des victimes, est-il besoin de rappeler que, jusqu'au début des années 1990, le mot « juif » n'était lisible nulle part, ni dans le musée, ni sur le monument international, à Auschwitz?

Il importe de commenter quelque peu la signification de cette situation absurde car ses enjeux étaient cruciaux et constituent une excellente introduction à l'étude des dimensions sociales et politiques de traumatismes collectifs, à ce que le philosophe Axel Honneth nomme justement « la grammaire morale des conflits sociaux» (Honneth, 1992).

Les Juifs parce que Juifs furent persécutés par une partie de l'humanité les nazis -, temporairement toute-puissante. Une autre partie de l'humanité - les Alliés - a combattu les nazis mais pas à cause des Juifs. Ils avaient des raisons indépendantes de le faire, et c'est presque exclusivement en fonction d'elles qu'ils ont conduit les opérations de guerre du début à la fin. Ne pas nommer les Juifs spécifiquement dans les hommages qui furent rendus après la guerre aux victimes du nazisme, c'était ignorer l'attaque particulière dont ils firent l'objet et, par conséquent, s'interdire de réparer les dommages particuliers qu'ils avaient subis. Quel est le sens d'un monument dédié aux victimes dans les ruines de Birkenau? C'est un jugement 
du monde qui a vaincu les nazis et qui - à l'emplacement même où ils mirent en pratique (et de quelle manière...) leur verdict déniant à certains toute appartenance à l'humanité - révoque ce verdict, le casse et réaffirme solennellement la pleine appartenance des victimes à l'humanité commune. Mais si, parmi ces victimes, il en est pour lesquelles les vainqueurs ne se sont pas battus et qui ne sont associées à l'hommage rendu que parce qu'il se trouve qu'elles étaient également ennemies de l'ennemi, alors l'hommage porte à faux. $\mathrm{Si}$, en outre, ces victimes représentent l'écrasante majorité et qu'elles ne sont présentes qu'à titre accessoire dans la commémoration, le malentendu vire au grotesque. Les Juifs - ceux qui l'étaient par conviction, par habitude ou par réaction - n'ont pas été dupes d'une célébration qui, précisément parce qu'elle ne les nommait pas, ne les réintégrait pas au sein de l'humanité dans la qualité au titre de laquelle ils en avaient été exclus. L'atteinte à leur dignité ne fut pas réparée : il ne fut pas établi qu'être Juif n'était pas infamant. Ici aussi, rarissimes furent alors les Juifs à réagir contre une situation qui perdura massivement en Europe, à l'Est comme à l'Ouest, plus d'un quart de siècle après la guerre. Parmi les exceptions, citons ce collaborateur du Bulletin du centre de documentation juive contemporaine dénonçant l'absence de toute mention spécifique des victimes juives dans une grande exposition consacrée aux «Crimes hitlériens » à Paris en 1945 :

C'est en tant que Juifs et non pas comme Roumains, Hongrois, Polonais, Tchécoslovaques ou Français que plus d'un million d'enfants, sans parler des autres, ont été amenés en Allemagne pour être enterrés vivants, gazés, vidés de leur sang, aux fins de la transfusion, ou affamés jusqu'à ce que mort s'ensuive. Paradoxe tragique que cette famille humaine contre laquelle s'acharnent si souvent, non seulement les ennemis des nations dont elle fait partie, mais ces nations elles-mêmes ; étrange sort de ces hommes, persécutés de leur vivant parce que Juifs, et que la mort seule fait admettre, sur un pied d'égalité, dans le bienheureux anonymat du paradis des Hollandais, des Polonais et autres Moldovalaques (Knout, $1945: 1$ ).

Était-il à ce point évident, comme beaucoup le prétendent aujourd'hui, que les Juifs avaient payé le plus lourd tribut à la folie meurtrière des nazis qu'il était inutile de le préciser explicitement? Sans doute pour certains, mais face aux jugements explicites des nazis, il aurait fallu un jugement aussi explicite de leurs adversaires. Ne pas l'avoir entendu fut perçu comme une trahison d'autant plus pénible qu'elle venait d'alliés et non d'ennemis. Comme, d'autre part, leur bannissement n'avait pas été un motif suffisant pour que le reste du monde prenne les 
armes, ils purent légitimement déduire que le reste du monde n'était pas en désaccord si total avec la sentence des nazis, que si ces derniers ne lui avaient donné d'autres motifs de mécontentement, il aurait pu s'accommoder - en le regrettant certes, mais sans le trouver intolérable - du sort réservé aux Juifs. Si, enfin, des indices donnent à suspecter que, même ici, le souci de ménager les antisémites constitue la raison tacite pour laquelle les Juifs ne sont pas nommés spécifiquement, comment ceux-ci peuvent-ils retrouver la moindre confiance dans les populations en lesquelles ils sont par ailleurs invités à se réintégrer le plus discrètement possible? Le lien social non plus n'a pas été restauré, moins encore réinstitué sur des bases nouvelles.

Non seulement cette reconnaissance élémentaire qui consiste à identifier les victimes fit défaut mais les Juifs furent blâmés pour la manière dont ils avaient réagi à leur sort. Tout comme, après un viol, les femmes furent longtemps stigmatisées de n'avoir pas su résister à leur agresseur, les masses juives assassinées se virent reprocher à titre posthume de s'être rendues à la mort « comme des moutons à l'abattoir ». Quant aux survivants juifs, leur situation était à peine moins défavorable : on les suspecta de s'être (plus) mal comportés (que d'autres) pour assurer leur survie. C'est selon moi dans cette double « victimisation secondaire »le blaming the victim syndrom - qu'il convient de chercher les racines profondes des conflits qui opposent aujourd'hui la plupart des milieux de mémoire juifs à d'autres groupes de persécutés. De façon plus immédiate, elle associa un sentiment durable de honte à la qualité de survivant de la Shoah alors même que la qualité d'ancien déporté résistant représentait au contraire un titre de gloire. Fortement intériorisée parce que les critères du jugement qui faisaient conclure à l'infamie étaient largement partagés au sein d'une communauté juive aspirant à la normalisation après la tourmente, la stigmatisation des victimes resta longtemps sans réponse adéquate : les rares milieux de mémoire juifs organisés tentèrent bien de faire valoir à l'époque l'insurrection dans le ghetto de Varsovie comme preuve de l'égale bravoure des Juifs mais la mise en relief de cette résistance ne faisait qu'accentuer, par son caractère exceptionnel, la prétendue « passivité » de tous ceux qui ne s'étaient pas insurgés ${ }^{1}$. Ainsi des milliers de personnes qui avaient été atteintes aux

1. Il serait trop long de mentionner ici l'ensemble des arguments historiques et moraux qui permettent de démonter le préjugé de la « passivité juive ». Je me permets de renvoyer sur ce point au septième chapitre (intitulé « Sordide, honte et grandeur : les mécanismes de la non-reconnaissance ») de mon livre (Chaumont, 1997) qui y est entièrement consacré. 
racines mêmes de la confiance, du respect et de l'estime de soi (Honneth, 1992) se virent privées des marques élémentaires de reconnaissance qui leur auraient permis de cicatriser autant que faire se peut les torts endurés. Il en résulta une humiliation et un ressentiment d'autant plus durables qu'ils restaient presque toujours tacites. Ils catalysèrent non seulement de nombreux traumatismes individuels mais affectèrent profondément la qualité du lien social entre les Juifs et les sociétés d'où ils avaient été déportés.

\section{Le retournement du stigmate}

Pourquoi est-il admis que nous pensions à l'Holocauste avec honte? Pourquoi ne le revendiquons nous pas comme un chapitre glorieux de notre histoire éternelle? [...] Peut-être que cela devrait être la tâche des éducateurs et des philosophes juifs : de rouvrir l'événement comme une source de fierté, de le reprendre dans notre histoire.

Elie Wiesel (1967 : 287).

Le propos d'Elie Wiesel cité ci-dessus illustre parfaitement la réaction tardive mais bien réelle que suscita finalement l'occultation du sort spécifique des Juifs et leur double stigmatisation après la guerre. Pas plus que la victimisation secondaire, ce retournement consistant à inverser la valence d'une expérience, soit en l'occurrence de la honte à la fierté, n'est sans précédent chez les groupes stigmatisés (Noël, 1989). Il prit cependant ici des formes particulièrement originales. Tout d'abord, répondant à l'injonction de Wiesel, des penseurs et des philosophes juifs se chargèrent effectivement de réinterpréter le comportement des victimes juives de telle sorte que l'un des plus influents dans le petit monde des Holocaust Studies a pu conclure par exemple que tous les Juifs sans exception méritaient le titre de résistants parce que, dans leur situation unique, vivre était devenu synonyme de résister (Fackenheim, 1982). D'autres s'occupèrent de renvoyer les reproches à leurs expéditeurs : ce n'était pas, dirent-ils, la passivité des Juifs qui était coupable mais bien au contraire la passivité du monde qui, indifférent, les abandonna à leur sort (Finkielkraut, 1980). Enfin, le fondement de la discrimination sociale que constitua la passivité alléguée des Juifs devint le fondement d'une singularité historique (soit le fait d'être persécuté pour ce que l'on est plutôt que pour ce que l'on a fait) qui prétendit justifier une distinction insigne : le monopole du statut de victime de «crime contre l'humanité ». Dans 
l'immédiat après-guerre, dans un pays comme la Belgique, les déportés juifs avaient dû se satisfaire d'un statut distinct de celui des déportés résistants : ces derniers seuls, qui pouvaient faire la preuve d'une " activité patriotique désintéressée », pouvaient arborer le titre glorieux de prisonniers politiques. Les autres ne pouvaient prétendre qu'au seul volet des réparations matérielles, à l'exclusion de la reconnaissance symbolique : ils étaient «bénéficiaires » et non «titulaires » du statut (Lagrou, 1989). Quarante ans plus tard, le renversement des rôles est complet : la base passée d'une disqualification à une reconnaissance publique officielle - n'avoir rien fait pour (défendre la patrie ou combattre le fascisme et donc) être déporté - est devenue la base présente de la qualification à un titre officiel alternatif et non moins envié qui est refusé aux résistants, c'est-à-dire aux concurrents de jadis : celui de victime du seul crime imprescriptible, le crime contre l'humanité. Désormais la victime est devenue le véritable héros et, tant en Europe qu'en Amérique, l'authentique témoin de la barbarie nazie n'est plus comme auparavant le militant politique de Buchenwald mais bien l'innocente victime d'Auschwitz. Ce sont à présent les résistants qui se sentent exclus de l'espace public et s'indignent qu'il faille rappeler dans les écoles que les Juifs ne furent pas les seuls à être persécutés par les nazis. Juste retour des choses que cette nouvelle hégémonie? Peut-être, sans doute... La revendication de l'unicité de la Shoab ne va cependant pas sans provoquer de redoutables effets pervers, c'est-à-dire des conséquences indésirables qui n'ont été voulues par personne mais avec lesquelles il faut bien compter. Je ne dirai rien ici des effets pervers des points de vue scientifique (pour l'intelligibilité du judéocide en particulier et du nazisme en général), juridique (pour l'élaboration des concepts de crime contre l'humanité ou de génocide), mémoriel (pour l'ancrage durable de la mémoire des crimes nazis dans la conscience occidentale) ou même identitaire (pour la reconstruction de l'identité juive contemporaine). Je me bornerai à évoquer l'effet pervers que je suppose le plus susceptible d'intéresser un public de victimologues : l'effet proprement politique que constitue la concurrence des victimes. 


\section{La concurrence des victimes}

Prétendre que l'Holocauste fut unique ne peut qu'impliquer que les tentatives pour annihiler d'autres

groupes nationaux ou culturels ne doivent pas être considérées comme des génocides, diminuant ainsi la

gravité et les implications morales de n'importe quel génocide, n'importe où, n'importe quand.

Cela implique aussi que les Juifs ont un monopole sur le génocide, que peu importe le malheur qui afflige un autre peuple, il ne peut être aussi sérieux ou même être compris dans une catégorie identique à l'Holocauste.

P. Papazian (1984 : 18).

Je ne me suis pas appesanti ici sur la manière dont la revendication de l'unicité absolue de la Shoab était devenue le fer de lance de l'entreprise de revalorisation des déportés juifs et, par extension, de l'identité collective juive. Arme de combat efficace pour forcer la reconnaissance tardive des autorités publiques et civiles, elle ne pouvait pas ne pas susciter l'hostilité d'autres groupes victimisés qui y virent une malveillante banalisation de leurs propres souffrances de la part « des » Juifs. Malveillante, elle ne l'était nullement puisqu'elle ne s'adressait même pas à eux ; mais banalisation indirecte, oui, indéniablement, et c'est précisément la raison pour laquelle de nombreux intellectuels juifs dénoncèrent très rapidement et très vigoureusement « l'obsession de l'unicité » (Schorch, 1981). Ainsi par exemple, Georges Steiner rétorqua sur le champ à Elie Wiesel en 1967 que «si tu interdis de comparer l'agonie de quelqu'un qui est torturé et brûlé vif maintenant à celle de ceux qui le furent alors et c'est, je pense, ce que tu es occupé à faire, Elie -, ceci est un fait que je crois infidèle au génie le plus profond de l'imagination juive et du sens juif de l'implication dans le destin de l'homme» (Steiner, 1967 : 289). Confinées pendant quelque temps dans des cercles restreints d'intellectuels juifs, les réactions ne tardèrent cependant pas à se manifester à l'extérieur : tant les autres groupes de victimes du nazisme (en particulier ces autres victimes « oubliées » que furent les Tziganes et les homosexuels) que d'autres victimes de l'histoire (en particulier les Arméniens, les Afro-Américains et les Amérindiens) contestèrent que la Shoab eût été à ce point singulière qu'elle doive être considérée comme absolument sans commune mesure avec ce qu'eux-mêmes avaient enduré par le passé. On assista alors à un véritable déplacement des lignes de front : des polémiques interminables, et malheureusement pas terminées, s'engagèrent 
et donnèrent lieu à des surenchères macabres dont presque tous, mais en en rejetant généralement la responsabilité sur la partie adverse, s'accordent à déplorer le caractère franchement indécent : concurrence des victimes pour la palme des plus grandes souffrances qui se produit sous le couvert d'un pseudo-débat historique.

L'unicité de la Shoab est ainsi devenue une pomme de discorde divisant farouchement des groupes minorisés qui pourraient plutôt, comme ce fut parfois le cas dans le passé, faire cause commune. Une source de conflits d'autant plus déplorable qu'elle ne permet même pas aux enjeux originels de reconnaissance - qui ne sont, eux, nullement sordides -, d'être publiquement débattus. Au contraire, des suspicions imaginaires, mais dont les conséquences sont bien réelles, foisonnent de part et d'autre. Les dialogues de sourds basés sur les malentendus ainsi générés sont particulièrement remarquables : tandis que « les » Juifs, abusivement recomposés en un groupe compact et homogène pour la circonstance, seront accusés par les autres de vouloir monopoliser le statut de victime, on soupçonnera à l'inverse chez les détracteurs de l'unicité un antisémitisme latent, voire une forme particulièrement perverse de négationnisme. Ni l'un ni l'autre de ces reproches ne sont fondés et chaque partie peut donc s'en défendre en toute bonne foi ; ce qui ne fait, bien sûr, qu'alimenter le cercle vicieux des récriminations réciproques.

\section{Conclusion en forme de question : d'où vient la valorisation des victimes?}

On doit se demander alors - et parvenu à ce point, j'ai plus de questions que de réponses -, quels sont les enjeux contemporains expliquant l'âpreté de la concurrence des victimes, des enjeux qui se sont greffés de façon conjoncturelle sur l'enjeu primaire qu'étaient les réactions aux dénis de reconnaissance de l'après-guerre. Observateur sévère mais bien informé de la scène sociale aux États-Unis, Todorov propose une hypothèse :

Si l'on parvient à établir de façon convaincante que tel groupe a été injustement traité dans le passé, cela lui ouvre dans le présent une « ligne de crédit » inépuisable. D'où la compétition effrénée pour obtenir, non comme entre pays la clause de la nation la plus défavorisée, mais celle du groupe le plus défavorisé (Todorov, 1995 : 99).

L'enjeu contemporain de la concurrence des victimes serait donc tout à fait classiquement un accès privilégié à des ressources matérielles et symboliques. Cette explication est certainement plausible mais elle 
appelle immédiatement une nouvelle question à laquelle j'espère que des victimologues pourront me suggérer des réponses : comment se fait-il que le statut de victime soit devenu tellement enviable?

Quelles que soient les erreurs dont puisse être entachée l'interprétation que j'ai suggérée, un fait me semble difficilement contestable (que je suis au demeurant loin d'être le seul à avoir pointé) : au cours des dernières décennies s'est produite dans l'aire de la civilisation occidentale une remarquable revalorisation du statut de victime. L'émergence même de la victimologie comme discipline autonome en est sans doute un des principaux symptômes. L'étude à laquelle je me suis livré illustre ce processus mais ne l'explique nullement. Or, il me parait clair que si elles n'avaient été en phase avec une tendance sociale lourde, les stratégies de revalorisation des victimes juives mises en œuvre par certains milieux de mémoire n'auraient pu aboutir aussi efficacement.

Considérons l'œuvre de philosophie politique majeure $\mathrm{du} \mathrm{Xx}^{\mathrm{e}}$ siècle aux États-Unis, la Théorie de la justice de John Rawls (1971). L'intuition morale fondamentale sur laquelle elle repose n'est-elle pas qu'il est juste d'être compensé pour les inégalités ou les désavantages que l'on subit? Autrement dit, le statut de victime représente la posture par excellence à partir de laquelle on peut exiger non seulement que justice soit faite mais que l'égalité entre les humains soit instaurée.

Or cette intuition morale n'a pas l'évidence que l'on peut être tenté de lui attribuer puisque force est bien de constater qu'elle est récente. Jusqu'il y a peu - et le culte des héros illustrait bien cette conception l'on jugeait plutôt que la justice exigeait que l'on traite chacun à l'aune de ses accomplissements. La question cruciale d'un point de vue sociologique est alors : comment est-on passé en quelques décennies, $d u$ point de vue des sensibilités morales collectives, d'une conception méritocratique (il est juste d'être rétribué pour ce que l'on fait) à une conception « victimaire » (il est juste d'être compensé pour ce que l'on subit) de la justice? On pourrait bien sûr rétorquer que cette compensation vise précisément à éliminer les inégalités héritées du passé et donc à établir les conditions d'une méritocratie non biaisée. Pourtant je ne crois pas qu'il suffise d'invoquer une dynamique autonome de la réflexion philosophique pour rendre compte de cette évolution. En effet, la sensibilité accrue au sort des victimes se manifeste également en d'autres champs sociaux - ceux en particulier qu'occupent la plupart des lecteurs de cette revue et qui les préoccupent - et il me semble que la justification de cette préoccupation relativement récente s'opère au nom d'argu- 
ments qui, tout en paraissant d'une évidence encore mieux partagée, n'en sont pas moins très différents parce qu'ils concernent une autre population (les victimes de faits qualifiés d'infractions) qui se trouve en butte à d'autres problèmes (les droits et l'accompagnement de la victime dans le processus judiciaire par exemple).

Il y a certes des échanges entre ces différents champs qui s'opèrent dans les discours et les pratiques. Ainsi le terme générique de survivors dont l'on baptise, dans le monde anglo-saxon, toutes les personnes affligées du syndrome post-traumatique, une affection à l'étude de laquelle ont notablement participé tant des spécialistes des traumatismes engendrés par la Shoab que d'autres se penchant sur ceux occasionnés par le viol (Herman, 1992). J'ai moi-même fait un usage du concept de « victimisation secondaire » en m'inspirant explicitement des productions de la victimologie. Néanmoins, on ne saurait contester que l'usage du concept de victime est bien large s'il peut également s'appliquer à la brave dame dont le sac a été arraché et au rescapé d'une chambre à gaz, à l'acteur d'un fait divers et à celui d'une tragédie de portée historique.

Et pourtant les uns et les autres font l'objet de la sollicitude du pouvoir. Aux uns, on demande pardon et on applique éventuellement la discrimination positive ; pour les autres, on crée des bureaux d'aide et des intervenants spécialisés. Les argumentaires sont différents, les mesures prises sont différentes mais toujours demeure, fragile plus petit commun dénominateur, l'invocation du statut de « victime ». Il est par conséquent plausible de faire l'hypothèse que tant le souci pour les victimes des crimes (collectifs) du passé que le souci pour les victimes des crimes (« privés ») du présent s'alimentent à une même source qui transcende la différence des argumentaires et des dispositions pratiques. Mais si on ne peut tout expliquer par les "progrès » de la réflexion morale ou juridique, comment rendre compte de la valorisation du statut de victime, où chercher, non plus les raisons mais les causes, de ce singulier phénomène? Des hypothèses partielles, relatives surtout à la promotion des victimes d'infractions, sont disponibles. Nul doute par exemple que les mouvements féministes ne puissent être crédités d'une bonne part de la reconnaissance accrue dont bénéficient enfin les victimes de viol de la part des autorités judiciaires et, de façon plus générale, dans la société civile. Dans d'autres cas, le souci proclamé des victimes apparait davantage comme l'instrument de combats conservateurs visant surtout au renforcement de la répression de la petite criminalité (Elias, 1993). Mais j'aurais tendance à croire que ces phénomènes 
localisés sont portés par des transformations sociales plus globales. Ainsi l'exclusion ou, pour reprendre le concept plus adéquat de Castel (1995), la désaffiliation est devenue un problème majeur de nos sociétés au cours des dernières décennies. Or les désaffiliés n'ont pas, comme l'avaient les exploités, la possibilité d'établir un rapport de force (en menaçant par exemple de paralyser l'appareil productif par la grève) pour négocier une position plus favorable dans le système. En effet, c'est précisément leur entrée dans le système qui est devenue problématique et à laquelle ils aspirent. Faute de réelle monnaie d'échange, quelle ressource leur restet-il sinon la posture de la victime innocente pour faire valoir leurs prétentions à l'insertion? Ce n'est qu'une hypothèse parmi d'autres possibles mais elle a le mérite de porter la question au cœur des fractures qui divisent nos sociétés et il me paraît que c'est bien à cet endroit que les configurations nouvelles s'esquissent. Cela étant, je ne puis mieux conclure qu'en invitant mes lecteurs victimologues à participer à la réflexion et au débat sur une problématique qui ne saurait les laisser indifférents.

\section{Références}

ANTELME, R. 1958. «Les principes à l'épreuve », Lignes janvier 1994 (21) : 113.

CASTEL, R. 1995. Les métamorphoses de la question sociale. Une chronique du salariat, Paris : Librairie Arthème Fayard.

Chaumont, J.-M. 1995. Connaissance ou reconnaissance? Les enjeux du débat sur la singularité de la Shoah, thèse de doctorat en sociologie, Paris : École des Hautes Études en Sciences Sociales.

Chaumont, J.-M. 1997. La concurrence des victimes. Génocide, identité, reconnaissance, Paris : Éditions La Découverte.

ELIAS, R. 1993. Victims Still. The Political Manipulation of Crime Victims, Newbury Park-Londres et New Delhi : Sage Publications.

FACKENHEIM, E. 1982. To Mend the World. Foundations of Future Jewish Thought, New York : Schocken Books.

FinkIELKRAUT, A. 1980. Le Juif imaginaire, Paris : Éditions du Seuil.

HERMAN, J. L. 1992. Trauma and Recovery. The Aftermath of Violence -from domestic abuse to political terror, s.l. : Basic Books (Une division de Harper Collins Publishers).

HONNETH, A. 1992. Kampf um Anerkennung. Zur moralischen Grammatik sozialer Konflikte, Francfort : Suhrkamp.

KLIEGER, B. 1946. Le chemin que nous avons fait... Reportages surbumains, Bruxelles : Éditions Beka.

KNOUT, D. 1945. « D'un mois à l'autre », Bulletin du centre de documentation juive contemporaine 1 (4) : 1. 
LAGROU, P. 1989. De politieke strijd om het verzetsaureool. Verdienste en enkentelijkheid in het na-oorlogste België (1944-1950), mémoire en histoire, Louvain : Katholieke Universiteit Leuven.

Lagrou, P. 1998. The Legacy of Nazi-Occupation. Patriotic Memory and National Recovery in Western Europe, 1945-1965, Cambridge : Cambridge University Press.

NoËL, L. 1989. L'intolérance. Une problématique générale, Québec : Les Éditions du Boréal.

PAPAZIAN, P. 1984. « A "Unique Uniqueness"? », Midstream 30 (4) : 14-18.

RAWLS, J. 1971. Theory of Justice, Cambrigde (Mass.) : Harvard University Press.

SARTRE, J. P. 1946. Réflexions sur la question juive, Paris : Paul Morihien.

SCHORSCH, I. 1981. "The Holocaust and Jewish Survival », Midstream 18 (1) : 38-42.

STEINER, G. 1967. « Jewish Values in the Post-Holocaust Future » (intervention orale dans le débat), Judaism 16 (3) : 266-299.

TODOROV, T. 1995. « Du culte de la différence à la sacralisation de la victime », Esprit (212) : 90-102.

WIESEL, E. 1967. « Jewish Values in the Post-Holocaust Future » (intervention orale dans le débat), Judaism 16 (3) : 266-299.

Wieviorka, A. 1992. Déportation et génocide. Entre la mémoire et l'oubli, Paris: Plon. 